\title{
Signal Traits and Oxidative Stress: A Comparative Study across Populations with Divergent Signals
}

\author{
Maren N. Vitousek ${ }^{1 *}$, Oldřich Tomášek ${ }^{2,3}$, Tomáš Albrecht ${ }^{2,3}$, Matthew R. Wilkins ${ }^{4,5}$ and \\ Rebecca J. Safran ${ }^{4}$ \\ ${ }^{1}$ Department of Ecology and Evolutionary Biology, Cornell University, Ithaca, NY, USA, ${ }^{2}$ Institute of Vertebrate Biology, The \\ Czech Academy of Sciences, Brno, Czech Republic, ${ }^{3}$ Department of Zoology, Faculty of Science, Charles University in \\ Prague, Prague, Czech Republic, ${ }^{4}$ Department of Ecology and Evolutionary Biology, University of Colorado Boulder, Boulder, \\ CO, USA, ${ }^{5}$ School of Biological Sciences, University of Nebraska, Lincoln, NE, USA
}

\section{OPEN ACCESS}

Edited by:

David Costantini,

University of Antwerp, Belgium

Reviewed by:

Andrea Bonisoli Alquati,

Louisiana State University, USA

Mathieu Giraudeau,

University of Exeter, UK

${ }^{*}$ Correspondence:

Maren N. Vitousek

mnv6@cornell.edu

Specialty section:

This article was submitted to

Behavioral and Evolutionary Ecology,

a section of the journal

Frontiers in Ecology and Evolution

Received: 16 December 2015 Accepted: 03 May 2016

Published: 19 May 2016

Citation:

Vitousek MN, Tomášek O, Albrecht T,

Wilkins MR and Safran RJ (2016)

Signal Traits and Oxidative Stress: A

Comparative Study across

Populations with Divergent Signals.

Front. Ecol. Evol. 4:56.

doi: 10.3389/fevo.2016.00056
Diverging populations often shift patterns of signal use-a process that can contribute to reproductive isolation and speciation. Yet it is not clear why most traits gain or lose signal value during divergence. One reason this could occur is because changes in the relationship between signals and relevant physiological parameters degrade the reliability of a signal, or even change its underlying information content. Here we test the hypothesis that the relationship between signal trait elaboration and a central component of organismal health-oxidative stress - differs across closely related populations that have diverged in signal use and preferences. In the recently diverged barn swallow subspecies complex (Hirundo rustica, Family: Hirundinidae), different populations use different traits as sexual signals. Two of these traits, ventral breast plumage color, and tail streamer length, differ markedly between North American H. r. erythrogaster and European H. r. rustica. Despite this divergence, variation in ventral plumage color was similarly associated with measures of oxidative damage across both populations. However, the directionality of these relationships differed between the sexes: darker male barn swallows had higher levels of plasma oxidative damage than their lighter counterparts, while the opposite relationship was seen in females. In contrast, relationships between tail streamer length and measures of oxidative stress were not consistent across populations. Some analyses indicated that in European H. r. rustica, where males bearing elongated streamers are preferred as mates, longer-streamered males were more oxidatively stressed; however, the opposite pattern was suggested in North American H. r. erythrogaster. Tail streamer length was not associated with measures of oxidative stress in females of either population. Differences in the physiological state of stronger signalers across populations and between the sexes may be related to costs or constraints on signal elaboration (e.g., biochemical pathways associated with melanogenesis), or reflect differences in how signal-mediated social interactions influence oxidative stress. Overall, our results suggest that while some phenotypic traits appear to be capable of conveying similar physiological information regardless of their use as signals, divergence in other phenotypic traits may be associated with shifts in their information content.

Keywords: sexual selection, social selection, speciation, physiology, antioxidants, barn swallows, Hirundo rustica 


\section{INTRODUCTION}

Signal traits are aspects of behavior, morphology, or physiology, that advertise information about how their bearer is likely to perform in a competitive context (Zahavi, 1975; Schluter and Price, 1993). Because the physiological state of an individual is often a crucial aspect of performance, signals that are causally linked with physiological measures can provide honest information about performance in relevant environmental and social contexts (Lailvaux and Irschick, 2006; Vitousek et al., 2014). Signal development and display can be associated with physiological state through many mechanisms. Some signals are costly to produce or display, so their elaboration may provide information about which individuals are able to bear these costs. As such, experimental manipulations have shown causal links between many aspects of physiological state and signal elaboration, including endocrine levels and the capacity to resist oxidative threats (Evans et al., 2000; McGraw et al., 2002; Blount et al., 2003; Alonso-Alvarez et al., 2004; Safran et al., 2008). Signals can also influence the physiological state of their bearer through social mechanisms (Rubenstein and Hauber, 2008; Oliveira, 2009; Vitousek et al., 2014). In situations where signals are tested (e.g., during competition), bearing a signal that does not equate with underlying competitive ability may result in "social persecution" that induces physiological costs (Rohwer, 1977; Tibbetts and Izzo, 2010). In contrast, if signals are honestly reflective of competitive ability, then social feedback about relative signal quality could induce positive changes in physiological state by altering hormone levels or other physiological mediators (Vitousek et al., 2013, 2014).

A central question in sexual selection and speciation concerns why populations diverge in signal phenotypes and preferences. If diverging populations are exposed to new environmental and social contexts, then shifts in signal use could result from different signals being most informative in these novel contexts (Schluter and Price, 1993). While substantial research has addressed the potential for signal efficacy to change across ecological contexts (Boughman, 2002; Tobias et al., 2010; Seehausen, 2015), less is known about how the information content of signals changes during divergence (Hebets and Papaj, 2005). Signal use could change because a different physiological state is optimal in different environments-and this information is most accurately conveyed by a different signal trait-or because the same optimal phenotypic state is best conveyed by different traits in each population. Alternatively, the physiological correlates of divergent phenotypic traits could differ in ways that are unrelated to their use as signals.

While many aspects of physiological state may play a role in sexual signaling, and in organismal function more broadly, oxidative stress is believed to be a particularly important element. Oxidative stress occurs when pro-oxidants-which can be generated by a number of oxidative processes including metabolism and immune activation-overwhelm antioxidant defenses and damage important biological macromolecules including DNA, proteins, and lipids (Costantini, 2008; McGraw et al., 2010; Metcalfe and Alonso-Alvarez, 2010). Oxidative stress can also impact senescence, at least in part through its effects on telomere dynamics (Von Zglinicki, 2002; Monaghan and Haussmann, 2006; Haussmann et al., 2011). Previous analyses have found that signal traits are both influenced by and can causally affect antioxidant capacity and oxidative damage (reviewed in Costantini, 2014).

We compared the relationship between diverging morphological traits and measures of oxidative stress in two closely related but recently diverged subspecies of barn swallows (Hirundo rustica, Family: Hirundinidae). Recently formed sister taxa are advantageous for comparative studies because, post-speciation, the process of divergence is confounded by further evolutionary changes (Via, 2001; Coyne and Orr, 2004). Recent mtDNA phylogeographic and microsatellite analyses in the barn swallow subspecies complex suggest that this group formed rapidly and is not strongly genetically differentiated, despite marked differentiation in two sexual signals: tail streamer length and the extent of ventral color (Dor et al., 2010). In North American barn swallows (H. r. erythrogaster) melaninbased ventral plumage coloration is a sexual signal; males with naturally darker or experimentally darkened plumage gain a higher proportion of paternity in their nests (Safran and McGraw, 2004; Safran et al., 2005). In contrast, while elongated tail streamers do not appear to be preferred by female H. $r$. erythrogaster (Safran and McGraw 2004), this trait is strongly sexually selected in European H. r. rustica (Møller, 1988). Males with elongated tails pair more quickly and have higher overall paternity levels (Saino et al., 1997a). Females in both subspecies display slightly reduced versions of the same signal traits used by males (Scordato and Safran, 2014). While the specific way in which ornamental traits are used by female barn swallows is not known, some evidence suggests that these traits may hold signal value. In $H$. r. erythrogaster darker females have greater reproductive success (Safran and McGraw, 2004), and both naturally darker and experimentally darkened females experience less oxidative damage (Vitousek et al., 2013). In H. $r$. rustica, streamer length appears to be under directional selection in females via increased reproductive success (Møller, 1993).

The specific signal traits used by barn swallows could be linked to oxidative stress in many ways. Melanin-based traits like the ventral plumage of barn swallows have been causally linked with oxidative stress in many species (McGraw, 2008; Galván and Alonso-Alvarez, 2009; Costantini, 2014); however, the direction of causation varies, and the mechanisms that generate these relationships are generally not well-resolved (Costantini, 2014). Melanin production may be influenced by oxidative stress, or by an allocation tradeoff between antioxidant defense and signal development (Jawor and Breitwisch, 2003; Moreno and Møller, 2006; Galván and Alonso-Alvarez, 2008; Metcalfe and AlonsoAlvarez, 2010). Melanin-based trait expression may also be coupled with oxidative stress levels through pleiotropic links between melanogenesis and the activity of the hypothalamicpituitary-adrenal (HPA) axis (Xiao et al., 2003; Ducrest et al., 2008; Jenkins et al., 2013). Depending on the specific pigment type produced (pheomelanins vs. eumelanins), these links may be positive (Galván and Solano, 2009; Galván et al., 2011) or negative (Almasi et al., 2010; Roulin and Ducrest, 2011). Plumage color could also affect oxidative stress levels through 
other mechanisms, including by influencing thermoregulatory capability (Sirkiä et al., 2010) or predation risk (Galván et al., 2014). Tail streamer length may also be linked with oxidative stress through several pathways. The ability to produce long tail streamers could be impacted by oxidative stress when plumage is replaced annually during seasonal molt. However, it is perhaps more likely that the direct energetic cost of bearing tail streamers longer or shorter than the aerodynamic optimum (Møller et al., 1995; Rowe et al., 2001) would excessively elevate pro-oxidant levels (Dowling and Simmons, 2009).

An alternative but not mutually-exclusive mechanism to explain potential links between signal traits and oxidative stress is that bearing exaggerated signals-regardless of the specific signal type-influences social interactions in ways that alter oxidative stress levels (Vitousek et al., 2013, 2014). For example, if stronger signalers are challenged more or less by conspecifics (Rohwer, 1985; Tibbetts and Dale, 2004), or if mates alter their provisioning investment based on signal quality (Dentressangle et al., 2008; Vitousek et al., 2014), then changes in signal elaboration could indirectly influence measures of oxidative stress. Finally, observed correlations between signal traits and oxidative stress may not be underlain by direct or indirect causal links.

To test whether differences in signal-physiology relationships among subspecies are associated with divergence in mate preferences, we assessed links between signal elaboration and two components of oxidative stress: plasma antioxidant capacity and oxidative damage (reactive oxygen metabolites), in both sexes of H. r. erythrogaster (breeding in Colorado, USA), and H. r. rustica (breeding in South Bohemia, Czech Republic). If the information content of signals is directly linked with trait type, then we would predict that the physiological correlates of each trait will be consistent across populations (Hypothesis 1: same trait, same information). If a different trait is a better indicator of a preferred physiological state in each population, then we would expect to see the same physiological state predicted by dark ventral coloration in North American H. r. erythrogaster males and by tail streamer length in European H. r. rustica males (Hypothesis 2: different traits, same information). If both signalphysiology relationships and the physiological state of preferred individuals differ across populations, then we would expect to find varying signal-physiology relationships across subspecies (Hypothesis 3: same trait, different information). Alternatively, signal traits could be unrelated to the measured physiological traits (Hypothesis 4: same trait, no information).

\section{METHODS}

\section{Capture and Sampling}

Barn swallows were captured with mist nets or by hand at breeding sites in Boulder and Jefferson Counties, Colorado, USA (CO; from May-July of 2010; 76 male and 83 female H. r. erythrogaster), and near Luznice, South Bohemia, Czech Republic (CZ; in May and June of 2011; 35 male and 27 female $H$. r. rustica). Blood samples were taken within $3 \mathrm{~min}$. of disturbance and placed on ice for several hours until centrifugation (10 $\mathrm{min}$ at $3500 \mathrm{rpm}$ ). Plasma was subsequently frozen at $-70^{\circ} \mathrm{C}$ until analysis. Body mass was measured with a Pesola spring balance (males and females in CO; males only in $\mathrm{CZ}$ ), and the right tail streamer measured to the nearest $\mathrm{mm}$. A sample of 4-6 breast feathers was plucked from the ventral surface, mounted on an index card, and stored in the dark until spectrophotometric analysis (Safran and McGraw, 2004). All capture and handling protocols were approved by the University of Colorado's Animal Care and Use Committee (IACUC \# SAF09-07-01), and by the Animal Care and Use Committees at the Czech Academy of Sciences (041/2011), and Charles University in Prague (4789/2008-30).

\section{Feather Color Measurements}

The color of melanin-based ventral breast plumage was scored using a reflectance spectrophotometer (Ocean Optics USB4000), according to previously described methods (Safran et al., 2010). Briefly, ambient light was excluded using a metal probe holder placed against the feather sample that ensured a constant distance from the probe to the sample. A fiber-optic probe with a PX2 pulsed xenon light source at an angle of $90^{\circ}$ to the feather surface generated reflectance data relative to a white standard (Ocean Optics WS-1) and a dark standard (for which all light was excluded). During each sampling period, 20 spectra were averaged with an integration period of $200 \mathrm{~ms}$. Each sample of breast feathers was scored three times, and average values were calculated. Previous analyses have indicated that ventral plumage brightness, a heritable trait in barn swallows (Hubbard et al., 2015), is highly correlated with hue and saturation in both populations (CO: Vitousek et al., 2013, CZ: Adámková, Albrecht, and Tomášek, unpublished data); we therefore used brightness alone in analyses.

\section{Analyses of Oxidative Damage and Antioxidant Capacity}

As a measure of oxidative damage, we used the d-ROMs kit (Diacron International, Grosseto, Italy) (Costantini et al., 2006, 2009) to assess the concentration of reactive oxygen metabolites (ROMs) - in this case primarily hydroperoxidesthat derive from the oxidation of biomolecules. In this test a chromogen mixture of alkyl-substituted aromatic amine reacts with metabolites, inducing a color change proportional to the concentration of metabolites. Plasma samples were added to $200 \mu \mathrm{L}$ of acetate buffer mixed with $2 \mu \mathrm{L}$ of chromogen $(N$, $N$-diethyl-p-phenylenediamine). After $75 \mathrm{~min}$. of incubation at $37^{\circ} \mathrm{C}$, the samples were centrifuged and $190 \mu \mathrm{L}$ of the supernatant was pipetted onto a microwell plate (Costantini et al., 2011b). The absorbance was read immediately at a wavelength of $505 \mathrm{~nm}$ (BioTek Synergy HT; VT, USA). Measured values were calibrated with a reference standard that substituted a calibrator solution of lyophilized serum for plasma, and converted to $\mathrm{mM}$ of $\mathrm{H}_{2} \mathrm{O}_{2}$ equivalents. Intra-assay variability was $7.9 \%$ and inter-assay variability was $6.7 \%$.

As a measure of antioxidant capacity, we estimated the total plasma antioxidant barrier (AOC) using the OXY-adsorbent test (Diacron International, Grosseto, Italy). This test quantifies the ability of plasma antioxidants (including proteins, ascorbate, 
thiols, vitamin E, and carotenoids) to resist oxidation by an endogenously produced oxidant, hypochlorous acid $(\mathrm{HOCl})$. Plasma samples were diluted 1:100 with distilled water, and a $200 \mu \mathrm{L}$ aliquot of $\mathrm{HOCl}$ solution was incubated with $5 \mu \mathrm{L}$ of the diluted plasma for $10 \mathrm{~min}$ at $37^{\circ} \mathrm{C}$ (Costantini et al., 2011b). Reference standards and blanks were prepared using $5 \mu \mathrm{L}$ of calibrator solution and water, respectively, and incubated with $200 \mu \mathrm{L}$ of $\mathrm{HOCl}$. At the end of the incubation, $5 \mu \mathrm{L}$ of chromogen solution ( $N, N$-diethyl- $p$-phenylenediamine) was added. The absorbance was read immediately at a wavelength of $505 \mathrm{~nm}$ (BioTek Synergy HT), and measured values (expressed in $\mathrm{mM}$ of $\mathrm{HOCl}$ neutralized per $\mathrm{mL}$ of sample) were calibrated with a reference standard that neutralized $350 \mathrm{mM}$ of $\mathrm{HOCl} / \mathrm{mL}$. Intra-assay variability was $5.6 \%$ and inter-assay variability was $8.9 \%$.

\section{Data Analyses}

Data were analyzed using SAS 9.4. General linear models were used to test the predictors of antioxidant capacity and reactive oxygen metabolites. Data from both populations were combined, but separate models were run for males and females. Initial models in males contained the fixed effects: population, body mass, corrected sampling date (see below), breast brightness, streamer length, population $\times$ breast brightness, and population $\times$ streamer length. Initial models for females contained the same factors, with the exclusion of body mass, which was not measured in $\mathrm{CZ}$ females. Fully parameterized models are available as Supplementary Material (Table S1). Final models were identified through backwards elimination of non-significant effects $(p>0.15)$, and are presented here. Data on reactive oxygen metabolites were reciprocally transformed, and model residuals were checked to ensure they conformed to the assumption of normality. Significant interactions between population and signal traits were investigated using separate linear regressions in each population.

Because sampling dates occurred at different times relative to the initiation of the breeding season in the two populations, and it was often not possible to determine the specific reproductive stage of each captured individual, corrected sampling dates were calculated for each individual by calculating the difference between the actual sampling date and the mean sampling date of individuals in that population. Thus, while this measure provides an indication of whether an individual was sampled relatively earlier or later than other individuals in its population, it does not provide information about the breeding stage of an individual that would enable us to assess true seasonal effects.

The data sets on oxidative damage and antioxidant capacity in male barn swallows each contained a single outlier. Because of the potential for these outliers to influence model outcomes, models were run twice: once with the full data set, and once with the single outlier excluded from the full model and throughout the process of backwards elimination. Models of antioxidant capacity run with and without the outlier (a male from $\mathrm{CO}$ with a $\mathrm{z}$-score of 3.4) did not differ qualitatively. Models of oxidative damage run with and without the outlier on this measure (a male from $\mathrm{CZ}$ with a $z$-score of 5.3) did differ. We present the results from both sets of models here.

\section{RESULTS}

\section{Divergent Signaling Phenotypes}

As expected, analyses of both traits indicated significant divergence between $H$. r. erythrogaster and $H$. r. rustica. North American H. r. erythrogaster males have significantly darker ventral breast coloration $(t=11.8$, $d f=40.4$, $p<0.0001)$, and shorter tail streamers $(t=11.1, d f=50.4$, $p<0.0001$ ), than European H. r. rustica males (Figure 1). Similar relationships were seen in females: H. r. erythrogaster females had darker ventral breast coloration $(t=9.4, d f=$ 38.3, $p<0.0001)$ and shorter tail streamers $(t=13.8$, $d f=36.0, p<0.0001)$ than their European counterparts (Figure 1).

\section{Male Signaling Phenotype and Measures of Oxidative Stress}

In male barn swallows, plasma antioxidant capacity was significantly predicted by corrected sampling date (males sampled earlier in the season had higher antioxidant capacity), and by an interaction between population and streamer length (Table 1, Table S1; final model all males: $F_{(5,100)}=$ 546.76, $n=105, p<0.0001$; final model outlier removed: $\left.F_{(5,99)}=619.28, n=105, p<0.0001\right)$. Population-specific regressions of significant interactions revealed that in North American H. r. erythrogaster, males with shorter streamers have a higher antioxidant capacity (outlier removed: $F_{1}=4.32$, $p=0.042$ ), whereas in European $H$. r. rustica streamer length does not predict antioxidant capacity $\left(F_{1}=0.14, p=0.714\right.$; Figure 2).

When all individuals are included in the analysis, oxidative damage was significantly predicted by breast brightness (Figure 3; higher in darker birds), and by the interaction between population and streamer length (Table 1, Table S1; final model: $\left.F_{(6,85)}=833.21, n=91, p<0.0001\right)$. Population-specific regressions indicated that in North American H. r. erythrogaster, streamer length was unrelated to oxidative damage (Figure 2; $F_{1}=2.32, p=0.133$ ), whereas European $H$. $r$. rustica males with longer streamers have greater oxidative damage $\left(F_{1}=4.37, p=\right.$ 0.045). However, when the single outlier is excluded, oxidative damage remains significantly higher in darker birds, but the interaction between streamer length and population is no longer significant [Table 1, Table S1; final model: $F_{(7,83)}=791.78$, $n=90, p<0.0001]$.

\section{Female Signaling Phenotype and Measures Of Oxidative Stress}

In female barn swallows, plasma antioxidant capacity was not significantly predicted by any of the morphological traits measured, but females measured earlier in the season had higher antioxidant capacity [Table 1; final model: $F_{(3,93)}=580.58$, $n=96, p<0.0001]$. The final model of oxidative damage includes breast brightness alone; darker females had significantly lower levels of oxidative damage in both populations [Table 1, Figure 3; final model: $\left.F_{(2,86)}=1650.11, n=88, p<0.0001\right]$. 

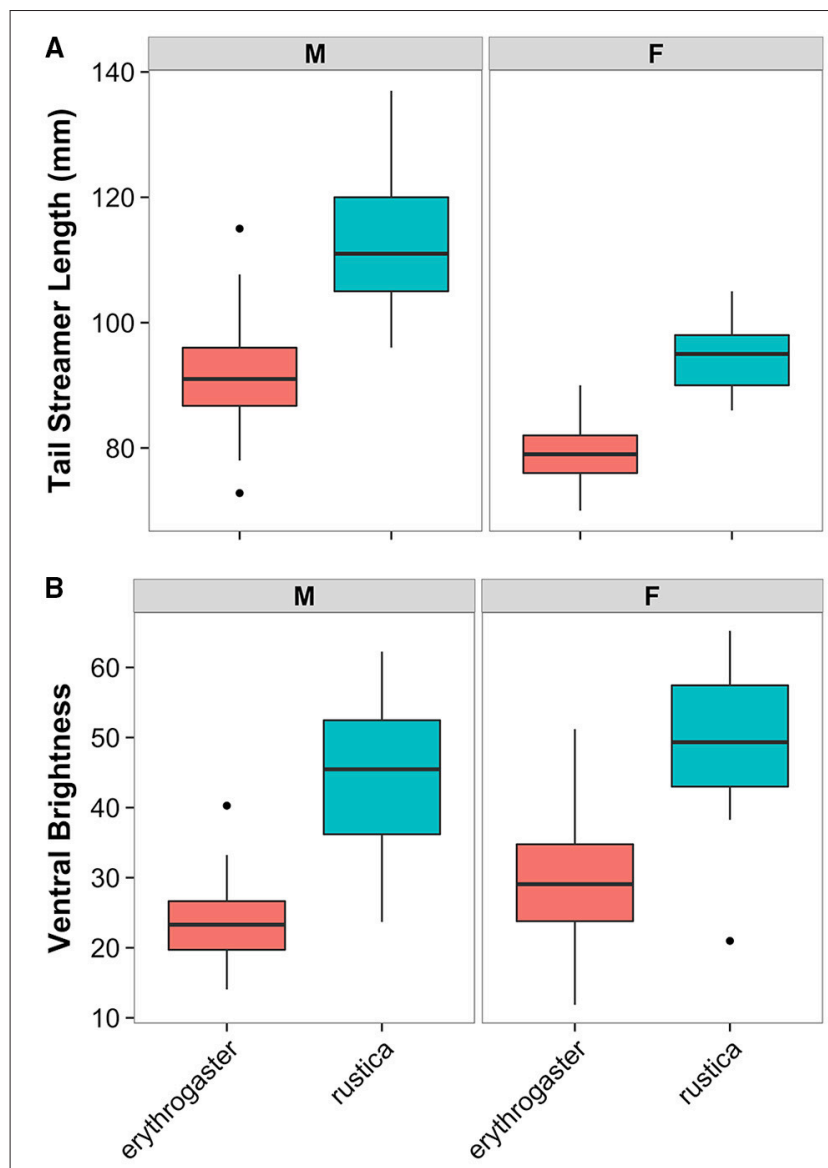

FIGURE 1 | The elaboration of sexual traits in divergent populations of barn swallows. (A) North American H. r. erythrogaster (red) have significantly shorter tail streamers than European H. r. rustica (blue) in both males and females. (B) H. r. erythrogaster of both sexes also have darker ventral breast plumage than H. r. rustica.

\section{DISCUSSION}

During divergence, shifts in signal use could occur because changes in signal-physiology relationships degrade signal reliability, or alter or eliminate its underlying information content (Schluter and Price, 1993). Yet while closely related species often differ in signal use, little is known about how the information content of signals changes during divergence. Our analyses indicate that in diverging populations of barn swallows, central components of physiological state (oxidative damage and plasma antioxidant capacity) are predicted by variation in the elaboration of two distinct plumage traits. While one of these signal traits (ventral color) is similarly associated with oxidative damage across populations, the other (tail streamer length) may not be.

The comparative approach utilized here does not enable us to determine whether signal traits are causally linked with measures of oxidative stress, or whether the observed relationships represent either a spurious relationship or result from both traits being uni-directionally influenced by a third unknown factor.
TABLE 1 | Final GLMs of antioxidant capacity and oxidative damage in male and female barn swallows.

\begin{tabular}{|c|c|c|c|}
\hline Model & Parameter Estimate & $\boldsymbol{F}$ & $\boldsymbol{P}$ \\
\hline \multicolumn{4}{|c|}{ ANTIOXIDANT CAPACITY: MALES } \\
\hline Population & 147.50 & 2.60 & 0.110 \\
\hline Corrected sampling date & -0.790 & 6.58 & 0.012 \\
\hline Streamer length & 0.328 & 2.19 & 0.142 \\
\hline Population*streamer length & -1.950 & 4.82 & 0.030 \\
\hline \multicolumn{4}{|c|}{ ANTIOXIDANT CAPACITY-OUTLIER REMOVED: MALES } \\
\hline Population & 136.55 & 2.51 & 0.116 \\
\hline Corrected sampling date & -0.651 & 4.96 & 0.028 \\
\hline Streamer length & 0.308 & 2.10 & 0.151 \\
\hline Population*streamer length & -1.815 & 4.68 & 0.033 \\
\hline \multicolumn{4}{|l|}{ OXIDATIVE DAMAGE: MALES } \\
\hline Population & 0.433 & 2.42 & 0.124 \\
\hline Corrected sampling date & 0.002 & 2.60 & 0.111 \\
\hline Streamer length & 0.003 & 0.00 & 0.952 \\
\hline Breast brightness & -0.005 & 7.91 & 0.006 \\
\hline Population*streamer length & -0.006 & 4.48 & 0.037 \\
\hline \multicolumn{4}{|c|}{ OXIDATIVE DAMAGE－OUTLIER REMOVED: MALES } \\
\hline Population & 0.435 & 2.11 & 0.150 \\
\hline Corrected sampling date & 0.002 & 4.39 & 0.039 \\
\hline Streamer length & 0.001 & 0.67 & 0.416 \\
\hline Breast brightness & -0.003 & 9.39 & 0.003 \\
\hline Population*streamer length & -0.004 & 2.13 & 0.148 \\
\hline Population*breast brightness & -0.006 & 3.18 & 0.078 \\
\hline \multicolumn{4}{|c|}{ ANTIOXIDANT CAPACITY: FEMALES } \\
\hline Corrected sampling date & -1.37 & 17.42 & $<0.001$ \\
\hline Streamer length & 0.901 & 2.42 & 0.123 \\
\hline \multicolumn{4}{|c|}{ OXIDATIVE DAMAGE: FEMALES } \\
\hline Breast brightness & 0.003 & 8.99 & 0.004 \\
\hline
\end{tabular}

Significant factors are bolded.

Previous experiments, however, have found that both plumage color and tail length causally influence measures of oxidative stress and other physiological traits in barn swallows (Saino et al., 1997b,c; Safran et al., 2008; Vitousek et al., 2013). Whether these causal links are present across trait types, populations, and the sexes is not known, and the specific mechanisms generating these links are not well-understood. The observational data presented here do, however, provide some insight into the mechanisms that could potentially generate these links.

\section{Plumage Brightness}

Ventral plumage color, a trait that is sexually selected in North American H. r. erythrogaster (Safran et al., 2005) but is not predictive of pairing success (clutch initiation date) in European H. r. rustica (Wilkins et al., unpublished data), is similarly associated with measures of oxidative stress in males of both populations. Males with darker ventral coloration have higher levels of oxidative damage, but do not differ in antioxidant capacity. Comparative data on plumage color signals in male barn swallows are therefore consistent with our first hypothesis: that the physiological correlates of a specific trait will remain constant across diverging populations (same trait, 

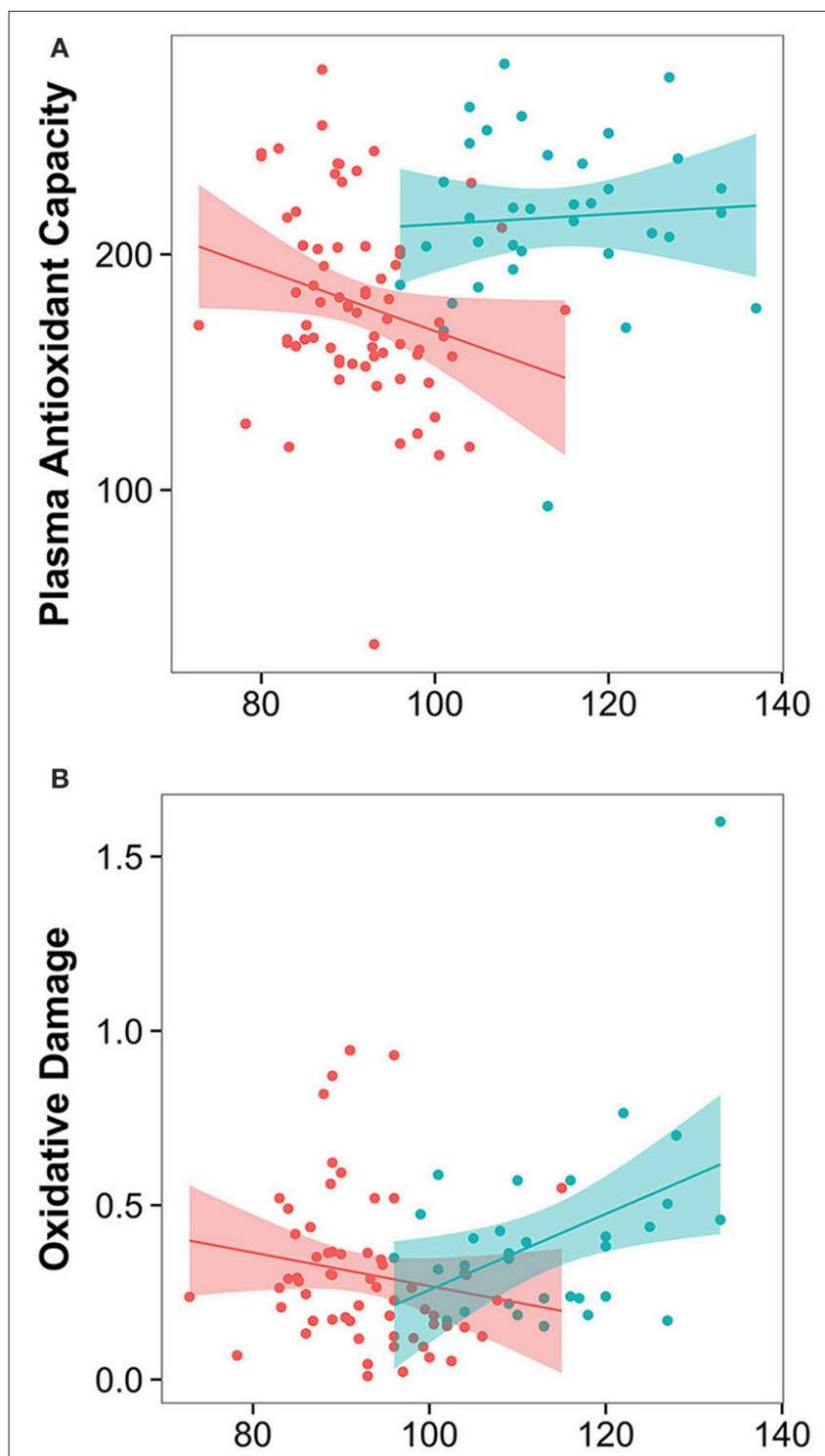

Tail Streamer Length ( $\mathrm{mm}$ )

FIGURE 2 | Male tail streamer length is differently associated with measures of oxidative stress in recently diverged populations that differ in signal use. Regressions of tail streamer length on: (A) Plasma antioxidant capacity ( $\mathrm{mM} \mathrm{HOCl}$ neutralized), and $\mathbf{( B )}$ oxidative damage ( $\mathrm{mM}$ $\mathrm{H}_{2} \mathrm{O}_{2}$ equivalents) in North American H. r. erythrogaster (red symbols and regression line) and European $H$. r. rustica (blue symbols and regression line).

Shading represents the $95 \%$ confidence interval for the linear regression.
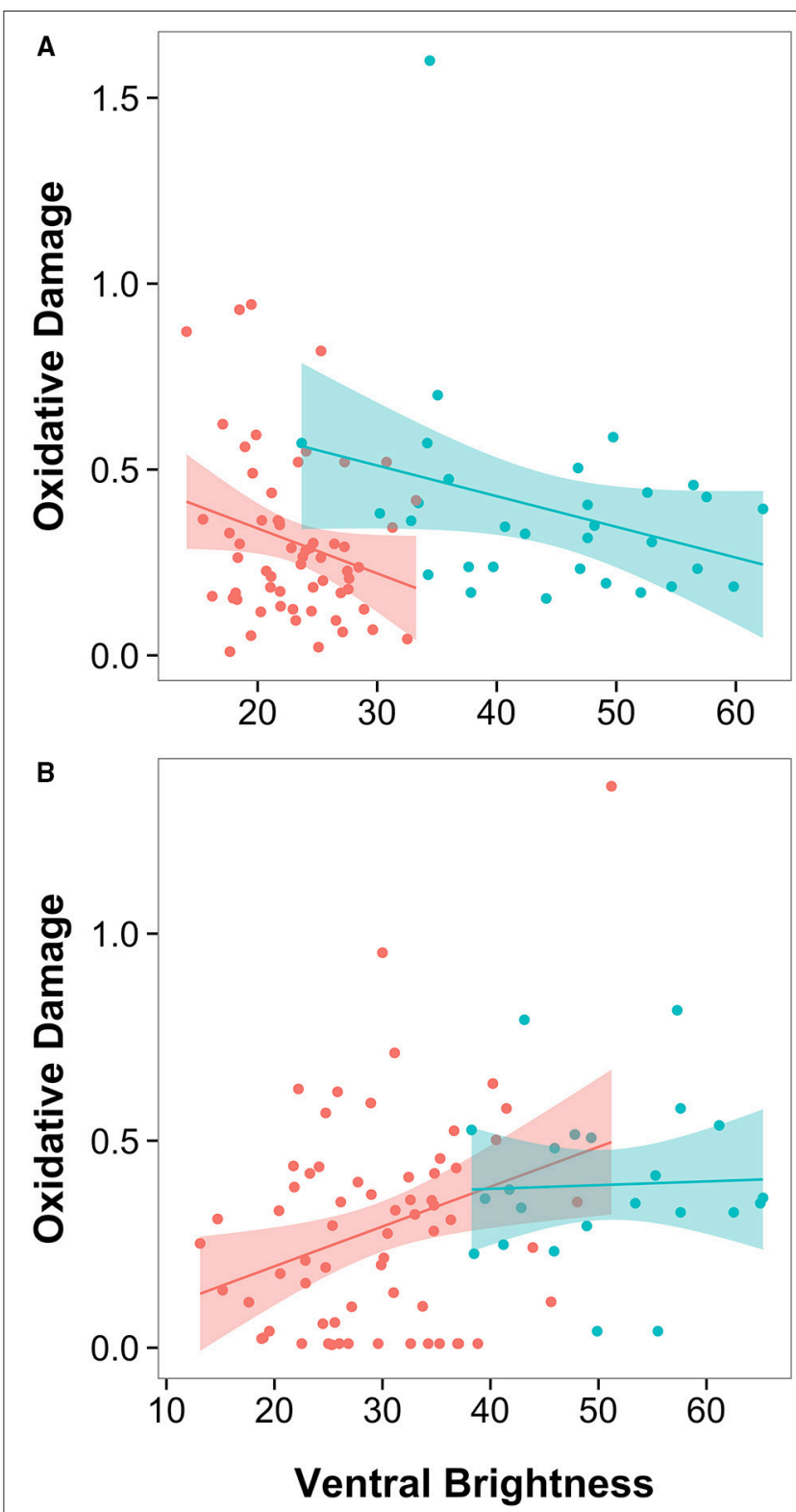

FIGURE 3 | Plumage brightness is oppositely related to plasma oxidative damage in male and female barn swallows: (A) Darker males (those with lower plumage brightness scores) have higher plasma oxidative damage ( $\mathrm{mM} \mathrm{H}_{2} \mathrm{O}_{2}$ equivalents) across both populations, (B) Darker females have lower oxidative damage. Simple regressions shown, with $H$. r. erythrogaster in red and H. r. rustica in blue. Shading represents the 95\% confidence interval for the linear regression. same information hypothesis). Intriguingly, while females also showed a consistent relationship between ventral plumage color and oxidative damage across populations, the direction of this relationship differed: in both North American H. r. erythrogaster and European H. r. rustica, darker females had less oxidative damage.

While we do not test the mechanisms underlying these relationships, the observed patterns provide some clues about the nature of the links between oxidative stress and plumage color. Because the plumage of these long-distance migrants is developed on the wintering grounds, long before the reproductive period, persistent links between signals and oxidative stress seem more likely to be driven by either fundamental constraints on system functionality (Hill, 2011), or by causal effects of trait display on oxidative stress (Vitousek et al., 2013). Similarly, the elevated levels of oxidative damage in 
darker male barn swallows is not consistent with trait elaboration providing information about the ability of an individual to resist challenges and withstand oxidative threats (von Schantz et al., 1999; Jawor and Breitwisch, 2003). The contrasting patterns found in males and females may, however, be consistent with the hypothesis that specific types of melanic pigments are differently linked with susceptibility to stress (Galván and Solano, 2015). While barn swallow plumage contains both eumelanins and pheomelanins, at least in H. r. erythrogaster, pheomelanin content appears to play a stronger role in male coloration than in female coloration (McGraw et al., 2005). Male ventral breast plumage contains more pheomelanins (but not eumelanins) than female plumage, and in males, but not females, the ratio of eumelanins to pheomelanins significantly predicts plumage coloration (McGraw et al., 2005). It is not known whether the sexes differ similarly in pigment composition in H. r. rustica, as the pigmentary basis of breast feathers has not been assessed (but see McGraw et al., 2004; Saino et al., 2013b).

Opposite relationships between these two types of melanic pigments and oxidative stress could occur through pleiotropic links between melanogenesis and the activity of the hypothalamic-pituitary-adrenal (HPA) axis-which often increases reactive oxygen metabolites (Haussmann and Marchetto, 2010; Costantini et al., 2011a). Pheomelanin production is influenced by agouti-related signaling protein, which also stimulates HPA axis activity (Xiao et al., 2003). Thus, darker pheomelanic individuals may be more sensitive to stressors (Galván and Alonso-Alvarez, 2011; Roulin and Ducrest, 2011; Saino et al., 2013a; Galván and Solano, 2015). In contrast, eumelanin pigment production is increased by melanin-stimulating hormone, which also binds to receptors in the hypothalamus that decrease HPA axis activity during the hormonal stress response (Racca et al., 2005; Ducrest et al., 2008). Thus, darker eumelanic individuals are predicted to display weaker hormonal stress responses (Almasi et al., 2010; Roulin and Ducrest, 2011) - and likely also lower levels of oxidative stress. Differences in the costs or trade-offs associated with producing these two pigment types could also be driven by other mechanisms. Pheomelanin production depends on the potent antioxidant glutathione; thus, the diversion of glutathione to pheomelanogenesis may be costly in organisms facing oxidative threats (Costantini, 2014). In contrast, eumelanogenesis can be inhibited by glutathione (Galván and Alonso-Alvarez, 2008, 2009; Hõrak et al., 2010). Thus, it is possible that, through any of several mechanisms, the consistent sex differences across populations in the directionality of the relationship between plumage brightness and oxidative stress are driven by differences in the costs or constraints of producing pheomelanic vs. eumelanic pigments.

Previous experiments in female $H$. r. erythrogaster, however, suggest that plumage coloration is causally linked with oxidative damage. Females manipulated to display darker plumage rapidly decrease plasma oxidative damage, adopting levels similar to naturally darker birds (Vitousek et al., 2013). While causal links between plumage color and oxidative damage have not been examined in males, or in $H$. r. rustica, erythrogaster males manipulated to display darker feathers rapidly decrease both testosterone and body mass (Safran et al., 2008), patterns that could be consistent with elevated levels of oxidative stress (Alonso-Alvarez et al., 2007; Costantini, 2014). Thus, at least in H. r. erythrogaster, plumage coloration appears to influence aspects of physiological state. The observed relationships between plumage color and oxidative damage in this study could be generated by consistent sex differences in the direct costs or benefits of displaying darker plumage-for example, if darker males suffer a thermoregulatory cost on the wing, while darker females are better able to retain heat during incubation (Sirkiä et al., 2010). Alternatively, plumage color may influence social interactions or reproductive effort in ways that alter oxidative stress (Vitousek et al., 2013, 2014). For example, if darker males invest more in costly reproductive behaviors this could increase their oxidative stress. This mechanism would be most likely to generate the observed patterns if, despite divergence of ventral coloration between the subspecies, this trait holds signal value in both populations. In H. r. rustica, ventral color, which is substantially lighter than in H. r. erythrogaster (Figure 1), does not predict the onset of breeding in males or females (Wilkins et al., unpublished data), as it does in H. r. erythrogaster (Safran and McGraw, 2004; Safran et al., 2005). However, it is not known whether in H. r. rustica, males with darker ventral color are preferred as extra-pair mates, or whether this trait plays a role in mediating other social interactions during or outside of the reproductive period. Experimental work in other systems has found that the value of the resource held by a signaler, or receiver motivation, can influence the likelihood that a signal will be tested (Tibbetts, 2008). If, during the breeding season, the plumage signals of male barn swallows are tested (e.g., during mate or nest site defense, or extra-pair mate assessment), while the signals of females are trusted, then we would predict that darker males would have higher levels of oxidative damage, while darker females would have lower oxidative damage-as seen here.

Previous analyses of the relationship between ventral color and susceptibility to stress have yielded mixed results. Ventral color was not related to HPA activity in H. r. erythrogaster males during molt or reproduction (Jenkins et al., 2013), suggesting that increased HPA activity in darker pheomelanic individuals is unlikely to drive the observed patterns with oxidative damage. Studies in a European population of H. r. rustica suggest that darker males-but not darker females-have lower survival rates (Saino et al., 2013a, but see Galván and Møller, 2013). It is not known, however, whether feather color is causally linked with survival in this population, and if so, whether this occurs through direct costs or constraints on pheomelanin production (Galván et al., 2011), or from physical or social costs of signal display.

Elucidating the direction and nature of causal links between ventral color and oxidative damage will require further experiments into the development and use of these traits within and across diverging populations. Additionally, as measures of oxidative stress can be highly labile, future studies that test 
whether the observed relationships differ across life history stages or environments, or represent context-dependent life history trade-offs (Beaulieu et al., 2015), could shed light on how and when diverging signals may convey specific, reliable information. Because we were not able to determine the reproductive sub-stage of individuals in the study, it is possible that differences between individuals, or between populations, in sample timing influenced the observed patterns. Future analyses that incorporate the specific reproductive stage of individuals-ideally as part of a repeated sampling designcould provide insight into the presence and nature of contextdependent links between signals and physiological state both within and among populations. In this analysis, we focused on a single region of ventral plumage color, the breast, as breast brightness has been shown to be strongly and causally associated with reproductive success in $H$. $r$. erythrogaster (Safran and McGraw, 2004; Safran et al., 2005). However, other plumage signals, including throat coloration, may hold signal value in H. r. rustica (Perrier et al., 2002; Wilkins et al., unpublished data). Some evidence also suggests that the relative roles of eumelanin- and pheomelanin-based pigments in determining plumage color differ between plumage patches (Saino et al., 2013b). Future analyses should be expanded to encompass other plumage traits that may hold signal value, and their pigmentary basis, and include additional key measures of physiological state and social interactions.

\section{Tail Streamer Length}

In contrast to the patterns observed with ventral color, comparative data on tail streamer length suggests that this trait is associated with different physiological information in males of these two subspecies. In European H. r. rustica, longer-streamered males did not differ in antioxidant capacity, but the analysis from the full data set indicated that they had greater levels of oxidative damage than shorter-streamered males. Thus, where tail streamer length is under positive sexual selection, males with longer streamers appear to experience higher levels of oxidative stress. In contrast, in North American H. r. erythrogaster, shorter-streamered males had a higher antioxidant capacity, but streamer length was not associated with oxidative damage. As plasma antioxidant levels are often up-regulated in response to oxidative threats, this pattern could indicate that shorter-streamered H. r. erythrogaster males are more oxidatively stressed (Costantini and Verhulst, 2009); however, this pattern could also result from naturally stronger antioxidant defenses in these males. Conclusions related to oxidative damage should be treated with caution due to the significant effect of the single outlier on the model outcomes. However, both models of antioxidant capacity (with and without the outlier) suggest that the physiological state of longer-streamered males differs across these diverging populations.

In male European barn swallows, $H$. $r$. rustica, sexual selection has resulted in the elongation of streamers past the aerodynamic optimum (Buchanan and Evans, 2000; Rowe et al., 2001). Barn swallows are aerial insectivores and so acquire all of their energetic resources during flight; thus, the apparent increase in measures of oxidative stress in longer-streamered $H . r$. rustica males could result from the increased energetic cost of foraging faced by these males. The lack of a relationship between tail streamer length and measures of oxidative stress in female $H$. r. rustica, who have shorter tail streamers than males (Figure 1), is consistent with females being closer to the aerodynamic optimum in this population. The elevated antioxidant levels in shorter-streamered North American $H$. $r$. erythrogaster males, which are consistent with elevated levels of oxidative stress, are particularly interesting. Previous analyses have indicated that short streamers could fall below the aerodynamic optimum for foraging flight (Buchanan and Evans, 2000; Rowe et al., 2001). Thus, shorter-streamered male H. r. erythrogaster-which appear to be preferred as mates by females (Safran et al., in revision) - could face increased foraging costs that translate into elevated oxidative stress. However, while female $H$. r. erythrogaster have even shorter tail streamers than their male counterparts (Figure 1), female tail length was unrelated to measures of oxidative stress. This could be because the direct costs of bearing shortened streamers differ in the sexes. For example, the increased wing-loading generated by elongated streamers might present a higher cost to shorterwinged females (Rowe et al., 2001), or differences in the foraging or reproductive behavior of female H. r. erythrogaster could select for shorter tail length. It is also possible that the opposing patterns in signal elaboration and physiological state in these two populations are influenced more by the social costs of trait display, or by signal-driven changes in reproductive effort, than by the direct effect of streamer length on aerial efficiency. For example, if tail length does not influence social status or social interactions in female barn swallows-but does in males-then we would expect to see social feedbackinduced links between oxidative stress and tail length in males (that differ in directionality between populations) but not in females.

\section{CONCLUSIONS}

Our analyses indicate that during the process of divergence, some morphological traits remain consistent indicators of oxidative damage, despite apparent shifts in signal use. Other traits, however, differ in their relationships with physiological state, and thus their potential information content, across diverging populations. While we did not directly assess the mechanisms that link signal traits and oxidative stress in barn swallows, our findings provided some clues about the nature of these links. As populations diverge, the same signals could be associated with different physiological states because of differences in the cost of producing or displaying an ornament across different physical environments (e.g., the same ornament is more costly to display in one environment than another), or because the same signal is differently elaborated in two populations (e.g., tail streamer length above or below the aerodynamic optimum: Rowe et al., 2001). Alternatively, divergent signal-physiology patterns could be a direct result of differences in the way signals are used across populations (e.g., social feedback about 
signal elaboration influences physiological state in populations where the trait is used as a social signal, but not where it is not). A comprehensive understanding of the relationship between signals and physiological state during divergence will require measuring a much wider variety of traits across multiple populations and contexts, in combination with experimental tests of the diversity of mechanisms that can generate these links. Our results do, however, suggest that the relationships between specific sexual traits and oxidative stress-a central component of organismal health-may be differently altered during divergence.

\section{AUTHOR CONTRIBUTIONS}

RS, MV, and TA conceived of the study. MV, OT, and TA collected field data. MV assayed oxidative damage and antioxidant capacity, analyzed the data, and drafted the manuscript. MW aided with analysis and interpretation. All authors contributed substantially to manuscript revisions, and approved the final version of the manuscript for submission.

\section{FUNDING}

Research funding was provided by the University of Colorado, and the Ministry of Education, Youth and Sports of the Czech Republic (project LH14045).

\section{ACKNOWLEDGMENTS}

We are grateful to Kathy Chmiel, Andrew Flynn, Kate Gloeckner, Joanna Hubbard, Tessa Warner, Rachel Wildrick, Jana Albrechtová, Jaroslav Cepák, Romana Michálková, Pavel Munclinger, and Martina Němcová for assistance with field work, and to Joanna Hubbard and Monica Brandhuber for measuring feather color.

\section{SUPPLEMENTARY MATERIAL}

The Supplementary Material for this article can be found online at: http://journal.frontiersin.org/article/10.3389/fevo. 2016.00056

\section{REFERENCES}

Almasi, B., Jenni, L., Jenni-Eiermann, S., and Roulin, A. (2010). Regulation of stress response is heritable and functionally linked to melanin-based coloration. J. Evol. Biol. 23, 987-996. doi: 10.1111/j.1420-9101.2010.01969.x

Alonso-Alvarez, C., Bertrand, S., Devevey, G., Gaillard, M., Prost, J., Faivre, B., et al. (2004). An experimental test of the dose-dependent effect of carotenoids and immune activation on sexual signals and antioxidant activity. Am. Nat. 164, 651-659. doi: 10.1086/424971

Alonso-Alvarez, C., Bertrand, S., Faivre, B., Chastel, O., and Sorci, G. (2007). Testosterone and oxidative stress: the oxidation handicap hypothesis. Proc. $R$. Soc. B Biol. Sci. 274, 819-825. doi: 10.1098/rspb.2006.3764

Beaulieu, M., Geiger, R. E., Reim, E., Zielke, L., and Fischer, K. (2015). Reproduction alters oxidative status when it is traded-off against longevity. Evolution 69, 1786-1796. doi: 10.1111/evo.12697

Blount, J. D., Metcalfe, N. B., Birkhead, T. R., and Surai, P. F. (2003). Carotenoid modulation of immune function and sexual attractiveness in zebra finches. Science 300, 125-127. doi: 10.1126/science. 1082142

Boughman, J. W. (2002). How sensory drive can promote speciation. Trends Ecol. Evol. 17, 571-577. doi: 10.1016/S0169-5347(02)02595-8

Buchanan, K. L., and Evans, M. R. (2000). The effect of tail streamer length on aerodynamic performance in the barn swallow. Behav. Ecol. 11, 228-238. doi: 10.1093/beheco/11.2.228

Costantini, D. (2008). Oxidative stress in ecology and evolution: lessons from avian studies. Ecol. Lett. 11, 1238-1251. doi: 10.1111/j.1461-0248.2008.01246.x

Costantini, D. (2014). Oxidative Stress and Hormesis in Evolutionary Ecology and Physiology. Berlin; Heidelberg: Springer Berlin Heidelberg.

Costantini, D., Casagrande, S., De Filippis, S., Brambilla, G., Fanfani, A., Tagliavini, J., et al. (2006). Correlates of oxidative stress in wild kestrel nestlings (Falco tinnunculus). J. Comp. Physiol. B 176, 329-337. doi: 10.1007/s00360-005-0055-6

Costantini, D., Dell'Omo, G., De Filippis, S. P., Marquez, C., Snell, H. L., Snell, H. M., et al. (2009). Temporal and spatial covariation of gender and oxidative stress in the Galápagos land iguana, Conolophus subcristatus. Physiol. Biochem. Zool. 82, 430-437. doi: 10.1086/604668

Costantini, D., Marasco, V., and Møller, A. P. (2011a). A meta-analysis of glucocorticoids as modulators of oxidative stress in vertebrates. J. Comp. Physiol. B. doi: 10.1007/s00360-011-0566-2

Costantini, D., Monaghan, P., and Metcalfe, N. B. (2011b). Biochemical integration of blood redox state in captive zebra finches (Taeniopygia guttata). J. Exp. Biol. 214, 1148-1152. doi: 10.1242/jeb.053496

Costantini, D., and Verhulst, S. (2009). Does high antioxidant capacity indicate low oxidative stress? Funct. Ecol. 23, 506-509. doi: 10.1111/j.13652435.2009.01546.x

Coyne, J. A., and Orr, H. A. (2004). Speciation. Sunderland, MA: Sinauer Associates.

Dentressangle, F., Boeck, L., and Torres, R. (2008). Maternal investment in eggs is affected by male feet color and breeding conditions in the blue-footed booby, Sula nebouxii. Behav. Ecol. Sociobiol. 62, 1899-1908. doi: 10.1007/s00265-0080620-6

Dor, R., Safran, R. J., Sheldon, F. H., Winkler, D. W., and Lovette, I. J. (2010). Phylogeny of the genus Hirundo and the Barn Swallow subspecies complex. Mol. Phylogenet. Evol. 56, 409-418. doi: 10.1016/j.ympev.2010. 02.008

Dowling, D. K., and Simmons, L. W. (2009). Reactive oxygen species as universal constraints in life-history evolution. Proc. R. Soc. B Biol. Sci. 276, 1737-1745. doi: $10.1098 /$ rspb.2008.1791

Ducrest, A., Keller, L., and Roulin, A. (2008). Pleiotropy in the melanocortin system, coloration and behavioural syndromes. Trends Ecol. Evol. 23, 502-510. doi: 10.1016/j.tree.2008.06.001

Evans, M. R., Goldsmith, A. R., and Norris, S. R. (2000). The effects of testosterone on antibody production and plumage coloration in male house sparrows (Passer domesticus). Behav. Ecol. Sociobiol. 47, 156-163. doi: $10.1007 /$ s002650050006

Galván, I., and Alonso-Alvarez, C. (2008). An Intracellular antioxidant determines the expression of a melanin-based signal in a bird. PLoS ONE 3:e3335. doi: 10.1371/journal.pone.0003335

Galván, I., and Alonso-Alvarez, C. (2009). The expression of melaninbased plumage is separately modulated by exogenous oxidative stress and a melanocortin. Proc. R. Soc. B Biol. Sci. 276, 3089-3097. doi: 10.1098/rspb.2009.0774

Galván, I., and Alonso-Alvarez, C. (2011). Natural radioactivity can explain clinal variation in the expression of melanin-based traits. Evol. Ecol. 25, 1197-1203. doi: 10.1007/s10682-011-9480-z

Galván, I., and Møller, A. P. (2013). Pheomelanin-based plumage coloration predicts survival rates in birds. Physiol. Biochem. Zool. 86, 184-192. doi: $10.1086 / 668871$

Galván, I., Mousseau, T. A., and Møller, A. P. (2011). Bird population declines due to radiation exposure at Chernobyl are stronger in species with pheomelaninbased coloration. Oecologia 165, 827-835. doi: 10.1007/s00442-0101860-5 
Galván, I., Nielsen, J. T., and Møller, A. P. (2014). Intensity of melanin-based color and risk of predation in the Barn Swallow Hirundo rustica. Acta Ornithol. 49, 47-56. doi: 10.3161/000164514X682887

Galván, I., and Solano, F. (2009). The evolution of eu- and pheomelanic traits may respond to an economy of pigments related to environmental oxidative stress. Pigment Cell Melanoma Res. 22, 339-342. doi: 10.1111/j.1755148X.2009.00559.x

Galván, I., and Solano, F. (2015). Melanin chemistry and the ecology of stress. Physiol. Biochem. Zool. 88, 352-355. doi: 10.1086/680362

Haussmann, M. F., Longenecker, A. S., Marchetto, N. M., Juliano, S. A., and Bowden, R. M. (2011). Embryonic exposure to corticosterone modifies the juvenile stress response, oxidative stress and telomere length. Proc. R. Soc. B Biol. Sci. 279, 1447-1456. doi: 10.1098/rspb.2011.1913

Haussmann, M. F., and Marchetto, N. M. (2010). Telomeres: Linking stress and survival, ecology and evolution. Curr. Zool. 56, 714-727.

Hebets, E. A., and Papaj, D. R. (2005). Complex signal function: developing a framework of testable hypotheses. Behav. Ecol. Sociobiol. 57, 197-214. doi: 10.1007/s00265-004-0865-7

Hill, G. E. (2011). Condition-dependent traits as signals of the functionality of vital cellular processes. Ecol. Lett. 14, 625-634. doi: 10.1111/j.14610248.2011.01622.x

Hõrak, P., Sild, E., Soomets, U., Sepp, T., and Kilk, K. (2010). Oxidative stress and information content of black and yellow plumage coloration: an experiment with greenfinches. J. Exp. Biol. 213, 2225-2233. doi: 10.1242/jeb.042085

Hubbard, J. K., Jenkins, B. R., and Safran, R. J. (2015). Quantitative genetics of plumage color: lifetime effects of early nest environment on a colorful sexual signal. Ecol. Evol. 5, 3436-3449. doi: 10.1002/ece3.1602

Jawor, J. M., and Breitwisch, R. (2003). Melanin ornaments, honesty, and sexual selection. Auk 120, 249. doi: 10.1642/00048038(2003)120[0249:MOHASS]2.0.CO;2

Jenkins, B. R., Vitousek, M. N., and Safran, R. J. (2013). Signaling stress? An analysis of phaeomelanin-based plumage color and individual corticosterone levels at two temporal scales in North American barn swallows, Hirundo rustica erythrogaster. Horm. Behav. 64, 665-672. doi: 10.1016/j.yhbeh.2013.08.006

Lailvaux, S. P., and Irschick, D. J. (2006). A functional perspective on sexual selection: insights and future prospects. Anim. Behav. 72, 263-273. doi: 10.1016/j.anbehav.2006.02.003

McGraw, K. J. (2008). An update on the honesty of melanin-based color signals in birds. Pigment Cell Melanoma Res. 21, 133-138. doi: 10.1111/j.1755148X.2008.00454.x

McGraw, K. J., Cohen, A. A., Costantini, D., and Hõrak, P. (2010). The ecological significance of antioxidants and oxidative stress: a marriage between mechanistic and functional perspectives. Funct. Ecol. 24, 947-949. doi: 10.1111/j.1365-2435.2010.01772.x

McGraw, K. J., Mackillop, E. A., Dale, J., and Hauber, M. E. (2002). Different colors reveal different information: how nutritional stress affects the expression of melanin-and structurally based ornamental plumage. J. Exp. Biol. 205, 3747-3755.

McGraw, K. J., Safran, R. J., Evans, M. R., and Wakamatsu, K. (2004). European barn swallows use melanin pigments to color their feathers brown. Behav. Ecol. 15, 889-891. doi: 10.1093/beheco/arh109

McGraw, K. J., Safran, R. J., and Wakamatsu, K. (2005). How feather colour reflects its melanin content. Funct. Ecol. 19, 816-821. doi: 10.1111/j.13652435.2005.01032.x

Metcalfe, N. B., and Alonso-Alvarez, C. (2010). Oxidative stress as a lifehistory constraint: the role of reactive oxygen species in shaping phenotypes from conception to death. Funct. Ecol. 24, 984-996. doi: 10.1111/j.13652435.2010.01750.x

Møller, A. P. (1988). Female choice selects for male sexual tail ornaments in the monogamous swallow. Nature 332, 640-642. doi: 10.1038/332640a0

Møller, A. P. (1993). Sexual selection in the Barn Swallow Hirundo rustica. III. Female tail ornaments. Evolution 47, 417. doi: 10.2307/2410061

Møller, A. P., De Lope, F., and Saino, N. (1995). Sexual selection in the barn swallow Hirundo rustica. VI. Aerodynamic adaptations. J. Evol. Biol. 8, 671-687. doi: 10.1046/j.1420-9101.1995.8060671.x

Monaghan, P., and Haussmann, M. F. (2006). Do telomere dynamics link lifestyle and lifespan? Trends Ecol. Evol. 21, 47-53. doi: 10.1016/j.tree.2005. 11.007
Moreno, J., and Møller, A. P. (2006). Are melanin ornaments signals of antioxidant and immune capacity in birds? Acta Zool. Sin. 51, 202-208.

Oliveira, R. F. (2009). Social behavior in context: hormonal modulation of behavioral plasticity and social competence. Integr. Comp. Biol. 49, 423-440. doi: 10.1093/icb/icp055

Perrier, C., de Lope, F., Møller, A. P., and Ninni, P. (2002). Structural coloration and sexual selection in the barn swallow Hirundo rustica. Behav. Ecol. 13, 728-736. doi: 10.1093/beheco/13.6.728

Racca, S., Spaccamiglio, A., Esculapio, P., Abbadessa, G., Cangemi, L., DiCarlo, F., et al. (2005). Effects of swim stress and $\alpha$-MSH acute pre-treatment on brain 5-HT transporter and corticosterone receptor. Pharmacol. Biochem. Behav. 81, 894-900. doi: 10.1016/j.pbb.2005.06.014

Rohwer, S. (1977). Status signaling in Harris' sparrows: some experiments in deception. Behaviour, 61, 107-129. doi: 10.1163/156853977X00504

Rohwer, S. (1985). Dyed birds achieve higher social status than controls in Harris' sparrows. Anim. Behav. 33, 1325-1331. doi: 10.1016/S0003-3472(85)80193-7

Roulin, A., and Ducrest, A.-L. (2011). Association between melanism, physiology and behaviour: a role for the melanocortin system. Eur. J. Pharmacol. 660 , 226-233. doi: 10.1016/j.ejphar.2011.01.036

Rowe, L. V., Evans, M. R., and Buchanan, K. L. (2001). The function and evolution of the tail streamer in hirundines. Behav. Ecol. 12, 157-163. doi: 10.1093/beheco/12.2.157

Rubenstein, D. R., and Hauber, M. E. (2008). Dynamic feedback between phenotype and physiology in sexually selected traits. Trends Ecol. Evol. 23, 655-658. doi: 10.1016/j.tree.2008.07.010

Safran, R. J., Adelman, J. S., McGraw, K. J., and Hau, M. (2008). Sexual signal exaggeration affects physiological state in male barn swallows. Curr. Biol. 18, R461-R462. doi: 10.1016/j.cub.2008.03.031

Safran, R. J., and McGraw, K. J. (2004). Plumage coloration, not length or symmetry of tail-streamers, is a sexually selected trait in North American barn swallows. Behav. Ecol. 15, 455-461. doi: 10.1093/beheco/arh035

Safran, R. J., McGraw, K. J., Wilkins, M. R., Hubbard, J. K., and Marling, J. (2010). Positive carotenoid balance correlates with greater reproductive performance in a wild bird. PLoS ONE 5:e9420. doi: 10.1371/journal.pone.00 09420

Safran, R. J., Neuman, C. R., McGraw, K. J., and Lovette, I. J. (2005). Dynamic paternity allocation as a function of male plumage color in Barn swallows. Science 309, 2210-2212. doi: 10.1126/science.1115090

Saino, N., Bolzern, A. M., and Møller, A. P. (1997b). Immunocompetence, ornamentation, and viability of male barn swallows (Hirundo rustica). Proc. Nat. Acad. Sci. U.S.A. 94, 549-552.

Saino, N., Cuervo, J. J., Krivacek, M., de Lope, F., and Møller, A. P. (1997c). Experimental manipulation of tail ornament size affects the hematocrit of male barn swallows (Hirundo rustica). Oecologia 110, 186-190.

Saino, N., Primmer, C. R., Ellegren, H., and Møller, A. P. (1997a). An experimental study of paternity and tail ornamentation in the Barn Swallow (Hirundo rustica). Evolution 51, 562. doi: 10.2307/2411128

Saino, N., Romano, M., Rubolini, D., Ambrosini, R., Caprioli, M., Milzani, A., et al. (2013a). Viability is associated with melanin-based coloration in the Barn Swallow (Hirundo rustica). PLOS ONE 8:e60426. doi: 10.1371/journal.pone.0060426

Saino, N., Romano, M., Rubolini, D., Teplitsky, C., Ambrosini, R., Caprioli, M., et al. (2013b). Sexual dimorphism in melanin pigmentation, feather coloration and its heritability in the Barn Swallow (Hirundo rustica). PLoS ONE 8:e58024. doi: 10.1371/journal.pone.0058024

Schluter, D., and Price, T. (1993). Honesty, perception and population divergence in sexually selected traits. Proc. R. Soc. B Biol. Sci. 253, 117-122. doi: 10.1098/rspb.1993.0089

Scordato, E. S., and Safran, R. J. (2014). Geographic variation in sexual selection and implications for speciation in the Barn Swallow. Avian Res. 5:8. doi: 10.1186/s40657-014-0008-4

Seehausen, O. (2015). Beauty varies with the light. Nature 521, 34-35. doi: $10.1038 / 521034 \mathrm{a}$

Sirkiä, P. M., Virolainen, M., and Laaksonen, T. (2010). Melanin coloration has temperature-dependent effects on breeding performance that may maintain phenotypic variation in a passerine bird: temperature-phenotype interactions on breeding. J. Evol. Biol. 23, 2385-2396. doi: 10.1111/j.1420-9101.2010. 02100.x 
Tibbetts, E. A. (2008). Resource value and the context dependence of receiver behaviour. Proc. R. Soc. B Biol. Sci. 275, 2201-2206. doi: 10.1098/rspb.2008.0477

Tibbetts, E. A., and Dale, J. (2004). A socially enforced signal of quality in paper wasp. Nature 432, 218-222. doi: 10.1038/nature02949

Tibbetts, E. A., and Izzo, A. (2010). Social punishment of dishonest signalers caused by mismatch between signal and behavior. Curr. Biol. 20, 1637-1640. doi: 10.1016/j.cub.2010.07.042

Tobias, J. A., Aben, J., Brumfield, R. T., Derryberry, E. P., Halfwerk, W., Slabekoorn, H., et al. (2010). Song diverence by sensory drive in Amazonian birds. Evolution 64, 2820-2839. doi: 10.1111/j.1558-5646.2010.01067.x

Via, S. (2001). Sympatric speciation in animals: the ugly duckling grows up. Trends Ecol. Evol. 16, 381-390. doi: 10.1016/S0169-5347(01)02188-7

Vitousek, M. N., Stewart, R. A., and Safran, R. J. (2013). Female plumage colour influences seasonal oxidative damage and testosterone profiles in a songbird. Biol. Lett. 9, 20130539. doi: 10.1098/rsbl.2013.0539

Vitousek, M. N., Zonana, D. M., and Safran, R. J. (2014). An integrative view of the signaling phenotype: dynamic links between signals, physiology, behavior and social context. Curr. Zool. 60, 739-754. doi: 10.1093/czoolo/60.6.739

von Schantz, T., Bensch, S., Grahn, M., Hasselquist, D., and Wittzell, H. (1999). Good genes, oxidative stress and condition-dependent sexual signals. Proc. $R$. Soc. B Biol. Sci. 266, 1-12. doi: 10.1098/rspb.1999.0597
Von Zglinicki, T. (2002). Oxidative stress shortens telomeres. Trends Biochem. Sci. 27, 339-344. doi: 10.1016/S0968-0004(02)02110-2

Xiao, E., Xia Zhang, L., Vulliémoz, N. R., Ferin, M., and Wardlaw, S. L. (2003). Agouti-related protein stimulates the hypothalamic-pituitaryadrenal (HPA) axis and enhances the HPA response to interleukin1 in the primate. Endocrinology 144, 1736-1741. doi: 10.1210/en.2002 220013

Zahavi, A. (1975). Mate selection-a selection for a handicap. J. Theor. Biol. 53, 205-214. doi: 10.1016/0022-5193(75)90111-3

Conflict of Interest Statement: The authors declare that the research was conducted in the absence of any commercial or financial relationships that could be construed as a potential conflict of interest.

Copyright (c) 2016 Vitousek, Tomášek, Albrecht, Wilkins and Safran. This is an open-access article distributed under the terms of the Creative Commons Attribution License (CC BY). The use, distribution or reproduction in other forums is permitted, provided the original author(s) or licensor are credited and that the original publication in this journal is cited, in accordance with accepted academic practice. No use, distribution or reproduction is permitted which does not comply with these terms. 